\title{
FS CMA TYPE BINARIES
}

\author{
Anatoly S. Miroshnichenko ${ }^{1}$ and Sergey V. Zharikov ${ }^{2}$
}

\begin{abstract}
FS CMa type stars is a group of $\sim 70$ objects formerly known as unclassified stars with the $\mathrm{B}[\mathrm{e}]$ phenomenon. Their very strong emission-line spectra in combination with a nearly main-sequence luminosity suggest the binary nature for them. They possess strong IR excesses due to radiation of circumstellar dust that implies a compact distribution probably in a circumbinary disk. Our long-term spectroscopic monitoring revealed neutral metal lines, which always include that of $\mathrm{Li}$ I $6708 \AA$, in the spectra of some FS CMa objects indicating the presence of a cool star. We present a summary of our results with a first overview of FS CMa type binaries and review possible implications for the nature and evolutionary status of the entire group.
\end{abstract}

\section{Introduction}

One of the first optical spectroscopic and near-IR photometric surveys conducted almost 40 years ago showed that $\sim 10 \%$ of $\sim 700$ hot emission-line stars exhibited - forbidden lines and strong IR excesses due to radiation of circumstellar dust (Allen \& Swings 1976). This unusual group was called "peculiar Be stars" or Bep stars to distinguish them from classical Be stars which showed smaller IR excesses due to radiation from circumstellar gas. Another name for the group, $\mathrm{B}[\mathrm{e}]$ stars, pro' posed by Conti (1976) only reflects the presence of forbidden lines and not of the IR excess, which is found in the majority of these objects. After $\sim 20$ years of ' studies, which were not intense due to expected heterogeneity of the group mem'bers, Lamers et al. (1998) concluded that the B[e] phenomenon is observed in four classes of stars with known evolutionary status: pre-main-sequence Herbig Ae/Be stars, symbiotic binaries, compact Planetary Nebulae, and some supergiants (e.g., $\eta$ Car). At the same time, 30 original group members were declared unclassified objects with the $\mathrm{B}[\mathrm{e}]$ phenomenon.

\footnotetext{
${ }^{1}$ Department of Physics and Astronomy, University of North Carolina at Greensboro, P.O. 26170, Greensboro, NC, 27402-6170

${ }^{2}$ Instituto de Astronomía, Universidad Nacional Autónoma de Mexico, Ensenada, Baja California, Mexico, 22800
} 
The main problems with the unclassified objects were lack of spectral lines from the B-type star atmosphere due to a strong line emission and circumstellar continuum radiation as well as sparse data that hampered definite classification. Their properties were critically evaluated by Miroshnichenko (2007), who summarized the main group features as follows: 1) early-B to early-A type optical continuum with strong emission lines of hydrogen, Fe II, [O I], and sometimes of [Fe II] and [O III] (absorption lines from the hot star atmosphere may be present as well, but they are frequently veiled by the circumstellar continuum); 2) a large IR excess that peaks at 10-30 $\mu \mathrm{m}$ and sharply decreases longward; 3 ) location outside of star-forming regions; and 4) a secondary companion which can be a fainter and cooler star or a degenerate object. Several possibilities for their evolutionary status were rejected, and the group was renamed to FS CMa type objects (after a prototype object with the $\mathrm{B}[\mathrm{e}]$ phenomenon, Swings 2006). In particular, the premain-sequence status was rejected due to their location out of star-forming regions and a steeper decrease of the mid- and far-IR flux with wavelength than that of young stars. Also the FS CMa objects are most likely not a kind of proto-Planetary Nebulae, which show much smaller near-IR excesses.

Below we address the current view of the FS CMa group as binary systems and show some recent results of our studies of individual objects.

\section{Nature of the FS CMa group and its current size}

The FS CMa group initially contained 23 objects from Allen \& Swings (1976). Seven objects remaining from the 30 unclassified ones still has an uncertain status due to lack of data. The group was enlarged by 10 objects found in the IRAS database by cross-identification with catalogs of optical positions (Miroshnichenko et al. 2007). Another 20 candidates were found by Miroshnichenko et al. (2011) in the Hamburg survey of emission-line stars (Kohoutek \& Wehmeyer 1999), which was cross-correlated with the NOMAD catalog (Zacharias et al. 2004). Currently $\sim 20$ more candidates found in NOMAD using several photometric criteria (e.g., Miroshnichenko et al. 2007) are being observed spectroscopically to confirm the presence of the $\mathrm{B}[\mathrm{e}]$ phenomenon, and the search continues.

Miroshnichenko (2008) found that the $\mathrm{H} \alpha$ emission in most FS CMa objects is over an order of magnitude stronger than that of classical Be stars of the same spectral type. This requires mass loss rates of $\dot{\mathrm{M}} \geq 10^{-7} \mathrm{M}_{\odot} \mathrm{yr}^{-1}$ considering they are single stars. Theory predicts such rates only for single supergiants with $\mathrm{L} \geq 10^{5} \mathrm{~L}_{\odot}$ (Vink et al. 2001). Therefore, it is natural to assume that FS CMa objects are binary systems which experienced mass transfer due to a Roche lobe overflow phase in at least one of the components. The mass transfer should be non-conservative to explain a large amount of circumstellar dust in the systems.

\section{Detected binaries among the FS CMa objects}

Although the binary scenario seems very likely, it has been uneasy to confirm binarity of the group objects. The most obvious one is MWC 623 with a composite 

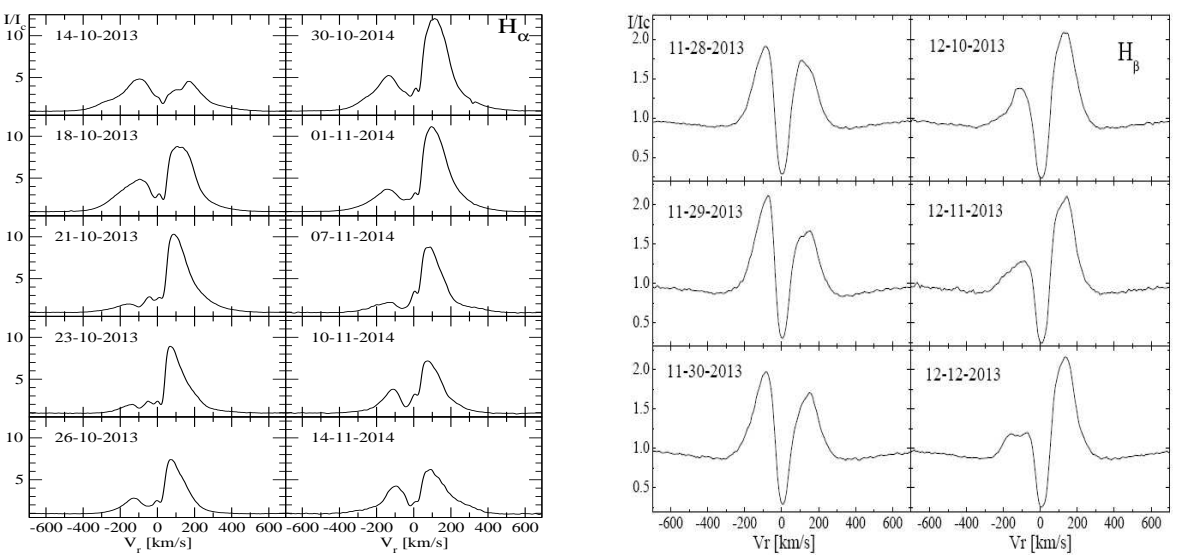

Fig. 1. Fast variations of the $\mathrm{H} \alpha$ line in the spectrum of MWC 728 (left panel) and of the $\mathrm{H} \beta$ line in the spectrum of HD 50138 (right panel). Observations were obtained at the Observatorio Astronomico Nacional San Pedro Martir (Baja California, Mexico) and the Three College Observatory (North Carolina, USA) with spectral resolving powers of $R=10000-18000$.

spectrum of a mid-B type primary and a $\mathrm{K}$-type secondary of a similar brightness (Zickgraf 2001). Much weaker signs of a composite spectrum have been detected in 10 other objects. Orbital periods have been measured only in two of them: CI Cam (the He II $4686 \AA$ line, 19.41 days, Barsukova et al. 2006) and MWC 728 (B5e+G8, metallic lines, 27.5 days, Miroshnichenko et al. 2015). The other 8 objects with a cool star lines, including that of $\mathrm{Li}$ I $6708 \AA$ whose origin in evolved stars is still under debate, are: V669 Cep (Miroshnichenko et al. 2002), FX Vel, AS 174, IRAS 00470+6429, IRAS 07080+0605, IRAS 17449+2320, IRAS 07377-2523 (Miroshnichenko et al. 2007), and AS 386 (Miroshnichenko et al. 2011). Binarity of the three brightest FS CMa objects (FS CMa, HD 50138, and HD 85567) was detected by spectro-astrometry (Baines et al. 2006), but neither spectroscopy nor interferometry have confirmed that yet.

\section{Other features and problems}

Our recent frequent spectroscopic observations of some FS CMa objects revealed significant variations of the Balmer line profiles on a timescale of days which do not seem to be regular (see Fig. 1). This implies variable mass loss from the hot component, although ongoing mass transfer due to a Roche lobe overflow has not been confirmed yet. These irregular variations may veil regular ones and hamper binarity confirmation. Nevertheless, the detected composite spectra and the measured orbital periods are consistent with non-conservative evolutionary models of binary systems (van Rensbergen et al. 2008). One of the future goals of the FSCMa group investigation is to search for objects at different stages of 
the binary evolution to identify when the dust formation begins and evaluate the group contribution to the Galactic dust production cycle.

A recent discovery of two FS CMa objects, which showed no signs of a cool secondary in the near-IR region, in Galactic clusters (de la Fuente et al. 2015) was interpreted as a consequence of binary mergers. Although this idea seems possible, the secondary may still be undetected due to its faintness with respect to the B-type primary and veiling by the circumstellar continuum. In any case, this result only enhances support for the binary nature of the FS CMa group.

\section{Acknowledgments}

We acknowledge support from DGAPA/PAPIIT project IN100614. AM acknowledges travel support from the University of North Carolina at Greensboro.

\section{References}

Allen, D.A., \& Swings, J. P. 1976, A\&A, 47, 293

Baines, D., Oudmaijer, R.D., Porter, J.M., \& Pozzo, M. 2006, MNRAS, 367, 737

Barsukova, E.A., Borisov, N.V., Burenkov, A.N., Klochkova, V.G., Goranskij, V.P., \& Metlova, N. V. 2006, in "Stars with the B/e] Phenomenon", eds. M. Kraus and A.S. Miroshnichenko, ASP Conf. Ser., 355, 305

Conti, P. 1976, in "Be and Shell Stars", ed. A. Slettebak, Proc. IAU Symp. 70, 447

de la Fuente, Najarro, F., Trombley, C., Davies, B., \& Figer, D.F. 2015, A\&A, 575, A10

Kohoutek, L., \& Wehmeyer, R. 1999, A\&AS, 134, 255

Lamers, H.J.G.L.M., Zickgraf, F.-J., de Winter, D., Houziaux, L., \& Zorec, J. 1998, A\&A, 340, 117

Miroshnichenko, A.S. 2007, ApJ, 667, 497

Miroshnichenko, A.S. 2008, in "Mass Loss from Stars and the Evolution of Stellar Clusters", eds. A. de Koter, L. J. Smith, and L. B. F. M. Waters, ASP Conf. Ser., 388, 205

Miroshnichenko, A.S., Bjorkman, K.S., Chentsov, E.L., et al. 2002, A\&A, 388, 563

Miroshnichenko, A.S., Manset, N., Kusakin, A.V., et al. 2007, ApJ, 671, 828

Miroshnichenko, A.S., Manset, N., Polcaro, V.F., Rossi, C., \& Zharikov, S.V. 2011, in "Active $O B$ stars: structure, evolution, mass loss and critical limits", eds. C. Neiner, G. Wade, G. Meynet, \& G. Peters, Proc. IAU Symp. 272, 260

Miroshnichenko, A.S., Zharikov, S.V., Danford, S., et al. 2015, ApJ, 809, 129

Swings, J.-P. 2006, in "Stars with the B[e] Phenomenon", eds. M. Kraus and A.S. Miroshnichenko, ASP Conf. Ser., 355, 3

van Rensbergen, W., De Greve, J.P., De Loore, C., \& Mennekens, N. 2008, A\&A, 487, 1129

Vink, J.S., de Koter, A., \& Lamers, H.J.G.L.M. 2001, A\&A, 369, 574

Zacharias, N., Monet, D.G., Levine, S.E., et al. 2004, BAAS, 36, 1418

Zickgraf, F.-J. 2001, A\&A, 375, 122 